Original Article

\title{
THERM OREGULATION IN THE HEART AND THE BIOPHYSICS OF CORONARY ARTERIAL FLOW
}

\author{
Arunachalam Kumar \\ Professor of Anatomy, K.S. Hegde Medical Academy, NITTE University \\ Mangalore - 575018 India \\ Correspondence: \\ Arunachalam Kumar \\ E-mail : editornujhs@nitte.edu.in
}

\begin{abstract}
:
The right and left coronary arteries are the principal sources of supply of oxygenated blood to the heart. Arising from the proximal rim of the ascending aorta, these arteries course along and over the surface of the organ, sending out branches that penetrate axial blood flow through arteries is governed or maintained by multifarious physical laws.

The heart, an electrically stimulated muscular pump, squirts and receives circulating blood through its systolic and diastolic exertions. The non-stop life-long rapid action of the organ not just expends enormous energy but also generates considerable heat. While there are a number of factors that help the heart dissipate and reduce heat, a few other biophysical factors contribute hugely to maintain a thermostatic milieu.

Circulation through vessels is maintained with a high degree of efficiency through combined actions of ejection systolic pressure, elasticity of the conducting arterial channels, and the proximo-distal decrease in diameters of arteries.

This brief write-up discusses some of physics regulatng fluid flow dynamics and attempts to exemplify the significant role of 'centrifugal force' as a hitherto overlooked physical force in coronary haemodynamics. The application of biophysical postulates to cardiac cycle may help in furthering our understanding of coronary blood circulation and the multi-factorial influences on its functional integrity. It is inferenced that, the finding may have a number of applications and result in a better understanding of cardiac circulatory dynamics.
\end{abstract}

Keywords: heart, biophysics, flapping coronaries, thermoregulation

\section{Introduction:}

The left and right coronary arteries are the principal sources of supply of oxygenated blood to the heart. Arising from the proximal rim of the ascending aorta, these arteries course along and over the surface of the organ, sending out branches that penetrate axially in to cardiac tissue.

Blood flow through arteries is governed or maintained by multifarious physical laws. The heart acting as an electrically stimulated

Access this article online Quick Response Code

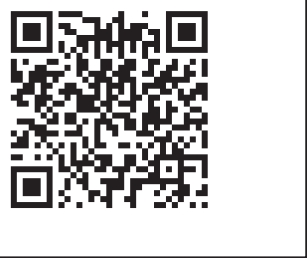
muscular pump, squirts out and receives circulating blood at its systolic and diastolic exertions. Circulation through vessels is maintained with a high degree of efficiency through combined actions of ejection systolic pressure, elasticity of the conducting arterial channels, and the procimo-distal decrease of diameters of arteries.

To better comprehend blood transportation, one needs to understand simple physical laws.The application of biophysical postulates to cardiac cycle may help in furthering our understanding of coronary blood circulation and the multi-factorial influences on its functional integrity.
This brief write-up discusses some areas of physics regulating fluid flow dynamics, and attempts to exemplify the undeniably significant role of 'centrifugal force' as a hitherto overlooked physical force in coronary haemodynamics. 


\section{Discussion:}

Canalized and channeled flow of liquids is not as simple a process as it first appears. The factors that allow channeled free flow of liquids from point ' $A$ ' to point ' $B$ ' are determined by many physical laws and principles. Some of the more readily obvious ones being:

\section{Gravity}

2.Atmospheric Pressure

3.Positive \& Negative pressures

4.Surface Tension

5.Viscosity

6. Inertia

Apart from these above cited physical dicta that determine fluid displacement from one point to another, an additional factors, the centrifugal force, could play an adjunct, yet vital role coronary arterial circulation.

Among the physical postulates that determine flow dynamics of fluids, Reyanuld's number, Re, is applied for calculations; it is the measure of the ratio of inertial forces to viscous forces and consequently quantifies the relative importance of these two types of forces for given flow conditions. Darcy - Weisbach friction factor used with Re gives an estimate of friction produced turbulence. Rate of flow through cylindrical pipes is determined applying the Hagen-Poiseuille equation.

Under ideal conditions (long, straight, smooth blood vessels), the critical Re is relatively However, in branching vessels, there can be turbulence even at normal physiological flow velocities. Turbulence generates sound waves that can be heard with a stethoscope. Because higher velocities enhance turbulence, murmurs intensify as flow increases. Elevated cardiac outputs, even across anatomically normal aortic valves, can cause physiological murmurs because of turbulence.

Coronary blood flow cannot be determined through application of any physical law directly: the vessels are elastic, tortuous and branch, each factor influencing flow dynamics. Moreover, laminar flow is constantly determined by mechanical, hormonal or neuronal systems that modulate the cardiovascular biomechanisms.

In some conditions, the fluid itself, blood, can and does change its viscosity and composition. These are factors too apart from pathological changes in anatomical structures too have a major impact on hemodynamics.

Considering that the organ functions at a frenetic pace, it should generate heat. There appears to be no specialized thermoregulatory mechanisms for the heart. Considering that the heart produces electrical impulses, contracts and relaxes seventy plus times a minute, controls the ejection and propulsion of nearly five liters of blood, it should be too 'hot' an organ. Yet, the heart maintains a steady ambient temperature! Notwithstanding the vortex, turbulence and friction of flow through coronary arteries, the heart remains pretty 'cool'. How it achieves this near impossible task has baffled biomedical engineers and biophysicists for long.

Of course, there a few pretty obvious heat-offsetting mechanismsin place;

1. The anatomical position of the heart itself, askew to the left and away from the sternum, its housing in a fenestrated bony cage with 'slats' (costae) and cardiac notch of left lung

2. The bathing of the organ in pericardial fluid

3. The location of the organ itself, smack in between two pneumonic sacs, the aerated lung.

4. While the anterior, right and left surfaces of the heart are related to the aerated lungs and intercostals space to dissipate heat, the posterior surface (base) which is related to the vertebral column and has no means to radiate heat - maintains heat through the cooling brought about by venous blood filled atrial chambers which form the posterior surface.

Each one of these factors may have a specific role in dissipating heat generated by the heart. This paper presents yet another, less studied in situ mechanism, a geometrical re-array of branching patterns in coronary arteries, especially in its proximal segments as another 
factor that probably reduces turbulence generated rise in cardiac temperature. Of late a few have described cardiac cycle as functioning chaotic systems. Chaos could also prove to be one of the non-linear mechanisms by which the heart stays thermostatic. (4)

In order to flow, any liquid blood inclusive, must utilize one or more of the above listed principles. While it is easy to decipher the application of all the above listed factors that govern motion, vis-a-vis blood circulation, 'centrifugal force' require some elaboration, especially so because it may a significant role in augmenting blood transport and delivery through the coronary arteries. (Figure 1)
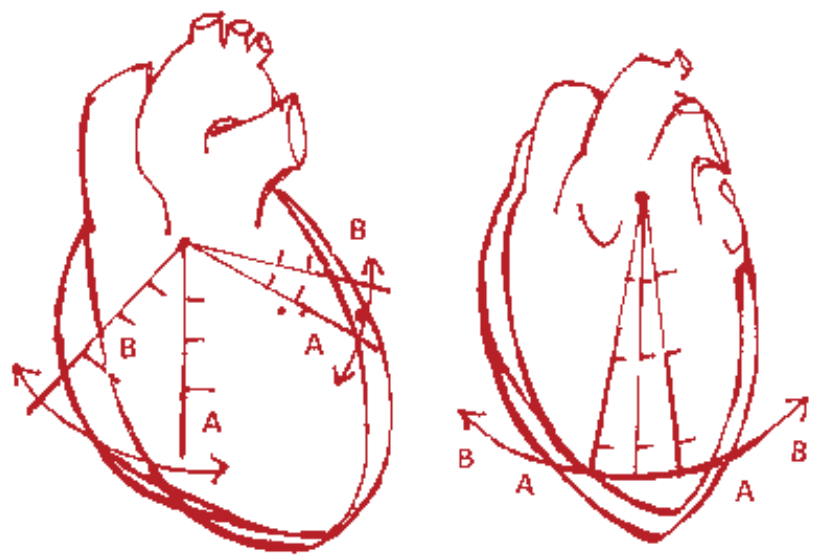

Figure 1: M ovements ( $\mathrm{A}-\mathrm{B}$ ) of coronary arteries during systole / diastole

Both coronary arteries appear as abruptly branching twigs from the main trunk of the proximal aorta. Their caudal descent on the surface of the atria, is angulated downwards and outwards. Their further course is a continuum of tortuousness, spiraling or tangential. Since these vessels run on the surface, with each systole-diastole unit of the cardiac cycle, the arteries, are perforce subjected to elevation- depression and abduction. The movements of the coronaries are oar-like, the aorticcoronary junction becoming the fulcrum. The pumphandle like arterial sorties engender centrifugal forces that are determined by application of the physical tenet: $m X v$ I $r$ which can be re-written as: $\mathrm{m} \mathrm{X} \mathrm{O}$ (where' $\mathrm{m}$ ' is the mass of the body, ${ }^{\prime} v$ ' is the velocity and ' $O$ is angular velocity)
The amplitudes and radii of the elevation-depression or abduction -adduction exercise of the arteries and the resultant angular velocity generated by the rapid whiplashing (flapping) generates centrifugal forces.The force thus created, more than seventy times a minute and with every contraction-relaxation cycle of the heart, acts as an adjunct biophysical factor in the transmission and delivery of blood the target tissue. (Figure 2)

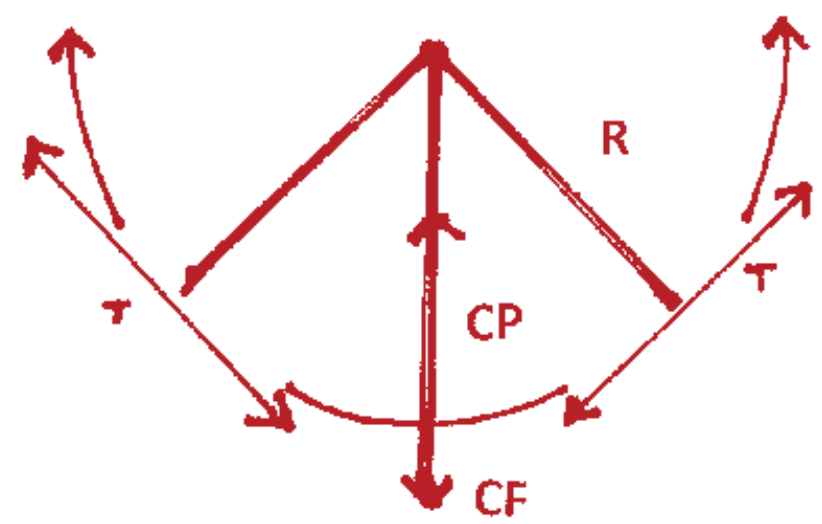

Figure 2: Diagram shows amplitude of movement of artery during systole / diastole (CP: centripetal force; CF: centrifugal force; T: tangential force; R: radius)

It is obvious that any change in amplitude of coronary movement, or in the radius along which it occurs will decrese or increase the centrifugal force. In other words, any change in dimensions of the heart, will not only alter the radius, but also the amplitude of the coronary swing arc influencing flow volume dynamics. The right artery is better equipped for generaton of centrifugal forces compared to the left: its direction is more rectilinear.

It is interesting to note that anatomically, all the early braches of the right coronary, run at right angles to the trunk, or tangential to the arc formed by the radius of the arterial segmental length. This right-angled braching system, allows a les turbulent and chaotic flow of the centrifugally ejected blood flow, which as per physical principles is tangential or at right angle to the parent arterial direction. Beyond the proximal segment, the right coronary, and alomost al the left, take a sinuous course, the centrifugal forces diminishing distally. Therefore, the branching pattern in the farther segments are acute angled or follow fractal orientation. 
What or how tachycardia or bradycardia effect centrifugal outflow, or how the coronary flow is effected by pericardoa; effusions, or hypertrophy of the heart ? Will any factor increasing the heart rate, by default, also raise the coronary arterial flagellation (whip-lash) rate?

\section{Conclusions:}

Observations presented here demonstrate that coronary flow is a complex dynamic system involving multiple physical and biomechanical principles and processes. The course of the artery on cardiac surface to allow free flow, the tortuousness course of its anatomy, its synergic interaction with myocardial contraction and relaxation and its unusual branching pattern ${ }^{1,2}$ at right angles in the proximal segment and bifurcating into acute angled branches in the more distal segment - all are factors that regulate the hemodynamics of coronary circulation (Figure 3).

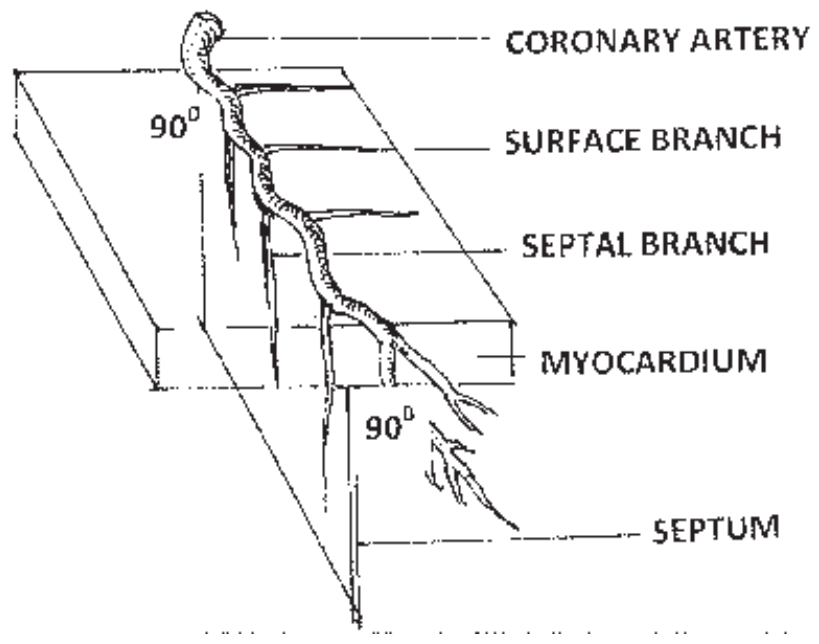

Figure 3: Coronary arterial course on surface of heart. Note right angled septal and surface branching from proximal segment

It is observed that physicians, cardiologists, physiologists have rudimentary knowledge on the physical principle regulating fluid motion. It is hoped that better understanding of cause and effect of compromise with the tenets of fluid mechanics, especially the role of inertia, centripetal, centrifugal and tangential forces $s^{4,5,6,7}$. Disturbance or disruption of laminar flow, either through mechanical or physiological causes, lead to turbulence and friction, can upset thermo regulatory mechanisms
The question raised is, if right- angled branching seen in proximal coronaries are inbuilt bio-regulators that counter or minimize turbulence then how would cardiac hypertrophy influence flow? Can the forced reduction of right to acute angle consequent to lengthening of parent coronary artery further compromise blood supply and flow to an already diseased heart?

There is little doubt that further research into this nebulous area is required. The combined input from biomechanical experts, fluid dynamic engineers and cardiologists could yield more information and understanding of this hitherto neglected area in hemodymics of coronary flow ${ }^{8}$

The purpose of this presentation is only to highlight a probably significant, but hitherto un-investigated biophysical mechanism that could act on coronary circulation haemodynamics. It is our conclusion that there may yet be many principles of biophysics waiting for application into the medical world.

Among the lesser understood, is the role of centrifugally generated forces and the modulation of the coronary arteries towards enhancing their effect on outflow. The arteries, more so the right coronary, bears a singularly unique branching pattern in that almost all named branches, course out at right angles to the trunk. That this pattern maximally utilizes the coronary outflow corroborates the theory that the coronary arterial trunks act as mobile radii generating rapidly alternating centrifugally directed forces. The left trunk, being more sinuous and less linear, and thus less centrifugal force generating potential, bears more branches that follow a more conventional acute angled fractal pattern.

What role, if any, does cardiac hypertrophy, resulting from a bacterial or viral endocarditis induced cardiomyopathy, have in altering angle of coronary branching ? Will the increase in lengths of proximal parts of the coronary tree that result in conversion of its right-angled branches to acute, and thereby compromise coronary blood flow dynamics in an already diseased heart?

In this brief hypothetical treatise on possible role of 
centrifugal forces, raises questions and maybe, stimulate further research into cardiac circulation and physiology, it would have more than amply served that purpose

\section{References}

1. Hutchins GM, Miner MM, Boitnott JK. ; Vessel caliber and branchangle of human coronary artery branch-points. Circ Res. 1976; 38(6):572-6.

2. Pflederer T, Ludwig J, Ropers D, Daniel WG, Achenbach S.; Measurement of coronary artery bifurcation angles by multidetector computed tomography Invest Radiol. 2006; 41(11):793-8.

3. Duncker DJ, Bache RJ. ; Regulation of coronary blood flow during exercise. Physiol Rev.2008; 88(3):1009-86

4. Kumar JC \& Arunachalam Kumar.; The biophysics and hemodynamics of coronary arterial flow. Proceedings of the $52^{\text {nd }}$ Annual Conference, A. S. I., 2004

5. Kumar JC \& Arunachalam Kumar.; Coronary arterial array and hemodynamics, Karnataka State Conference of the Cardiological Society of India, 2005

6. Kumar JC \& Arunachalam Kumar; Coronary arterial geometry and cardiac circulation, Proceedings of the Indo-Australian Conference, Manipal, 2005

7. Kumar $\mathrm{JC} \&$ Arunachalam Kumar; The physiodynamics and biomechanics of cardiac apex. BMJ (S. Asian Ed) 20; 3; 19-20, 2004

8. Arunachalam Kumar \& Hegde BM.; Chaos Theory: Impact on and Applications in Medicine. Nitte University Journal of Health Science 2012, 2 (4) 93-99 